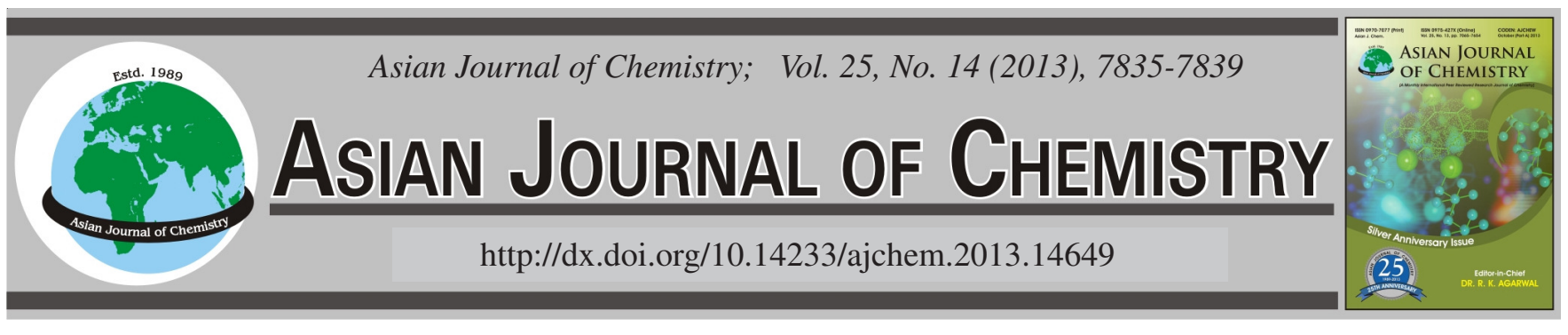

\title{
Synthesis and Flocculation Properties of Star-Shaped Cationic Polyacrylamide
}

\author{
Weimin Sun*, Guangcheng Zhang, Heng CaO and Helin Li
}

Department of Applied Chemistry, School of Science, Northwestern Polytechnical University, Xi'an 710072, P.R. China

*Corresponding author: Tel: +86 15829048689, E-mail: outcast191@163.com

(Received: 8 October 2012;

Accepted: 20 July 2013)

AJC-13821

\begin{abstract}
Star-shaped cationic polyacrylamide was synthesized using free-radical polymerization of acrylamide and acryloxyethyltrimethyl ammonium chloride (DAC). The co-polymerization of acrylamide and acryloxyethyltrimethyl ammonium chloride was conducted using pentaerythritol as a core and ammonium persulfate and sodium sulfite as the reduction oxidation initiation system. The effect of initiator dosages, monomer concentration, reaction temperature and the mass ratio of acryloxyethyltrimethyl ammonium chloride to acrylamide on polymerization were evaluated. Polymer product was characterized using Fourier-transform infrared spectroscopy and nuclear magnetic resonance spectroscopy. Flocculating properties were evaluated using the rates of chemical oxygen demand removal from sewage in standard jar tests. The polymer product functioned as a novel cationic flocculant in water and wastewater treatment.
\end{abstract}

Key Words: Star-shaped cationic polyacrylamide, Cationic polymeric flocculants, Coagulation and flocculation.

\section{INTRODUCTION}

Coagulation-flocculation is used widely during water or wastewater treatment ${ }^{1}$. Flocculants play an important role in this process. Most polymers used are acrylamide-based nonionic, anionic and cationic homopolymers or copolymers ${ }^{2}$. Cationic polyacrylamides are the most interesting ones from the commercial point of view, due to their higher added value ${ }^{3}$. High molecular weight linear polyacrylamide can be used as a polymer flocculant, but in aqueous solution has a very high viscosity which is limiting ${ }^{4}$. Star-shaped polymers have been used for a long time. Compared with linear polymers, threedimensional star-shaped polymers have high solubility and low viscosity $^{5}$. These advantages led to a new design idea with a broad application in the modification of traditional polymers.

A star-shaped cationic polyacrylamide was synthesized using pentaerythritol as the core, acrylamide and acryloxyethyltrimethyl ammonium chloride (DAC) as monomers and ammonium persulfate and sodium sulfite as the reduction oxidation initiation system. The star-shaped cationic copolymer was characterized using Fourier-transform infrared spectroscopy and nuclear magnetic resonance spectroscopy. Flocculating properties were evaluated using chemical oxygen demand removal from sewage in standard jar tests.

\section{EXPERIMENTAL}

Acryloxyethyltrimethyl ammonium chloride (DAC, industrial grade), acrylamide, ammonium persulfate, sodium sulfite, pentaerythritol, sodium chloride, ferrous ammonium sulphate, potassium dichromate, silver sulfate, mercury sulfate, acetone and absolutely alcohol were all of analytical reagent grade.

The star-shaped cationic polyacrylamide was synthesized from acrylamide and DAC monomers in an aqueous solution. Acrylamide and distilled water were added to a $250 \mathrm{~mL}$ threenecked flask equipped with a stirrer and a thermometer. The solution was bubbled with nitrogen for $30 \mathrm{~min}$ to remove oxygen. The monomer DAC, pentaerythritol (PETL-the core of the polymer) and the initiators ammonium persulfate and $\mathrm{Na}_{2} \mathrm{SO}_{3}$ were added and the flask was then placed in a water bath at the reaction temperature, for the specified time. At the end of polymerization, the polymer was precipitated, washed with acetone and absolutely alcohol in turn, cut into small pieces and dried in a vacuum oven at $50{ }^{\circ} \mathrm{C}$ until a constant weight was obtained.

The Fourier transform infrared spectroscopy was measured using a Necolet IS10 FTIR spectrometer (USA). Samples were pressed into $\mathrm{KBr}$ pellets. ${ }^{1} \mathrm{H}$ NMR spectroscopy was measured using an INOVA-400 MHz NMR (Varian Co., USA) spectrometer at room temperature, with $\mathrm{D}_{2} \mathrm{O}$ as solvent. Intrinsic viscosity measurements were made using an Ubbelohde viscometer, with a sodium chloride solution as the solvent.

Flocculation properties of the star-shaped cationic polyacrylamide were evaluated using the rates of chemical oxygen demand (COD) of sewage determined from jar tests. The sewage was obtained from the wastewater treatment plant of 
Chang' an campus, Northwestern Polytechnical University. All coagulation experiments were conducted in $1 \mathrm{~L}$ plexiglass beakers, using a ZR4-6 coagulation test stirrer (Shenzhen Zhongrun water industrial technology development Co., Shenzhen, China). $1000 \mathrm{~mL}$ water sample solutions were added with different coagulants. The solution was stirred at $400 \mathrm{rpm}$ for $1 \mathrm{~min}$ during coagulant addition, followed by $10 \mathrm{~min}$ at 40 rpm and 15 min sedimentation. After sedimentation, a supernatant sample was withdrawn from a point $3 \mathrm{~cm}$ below the surface of the test water sample, for COD measurement. COD was measured according to Chinese national standard methods ${ }^{6}$.

\section{RESULTS AND DISCUSSION}

Reaction mechanism: The molecular structure of pentaerythritol contains four $-\mathrm{CH}_{2} \mathrm{OH}$ groups, which can undergo a reduction oxidation reaction easily. Its space orientation and structure is completely symmetrical, making it suitable for forming branched polymers. The hydroxyl component can be coupled with an oxidant, forming a reduction oxidation initiator system. Once initiated, vinyl monomers are copolymerized onto the core unit. Ammonium persulfate is an oxidant with the ability to take up electrons when it meets a reducing agent. When ammonium persulfate meets pentaerythritol (which contains four hydroxyl groups), the free radical of pentaerythritol is formed. This initiated form of the star-shaped structure copolymer is the basis for further polymerization with acrylamide and DAC. The reaction scheme is shown in Fig. 1.

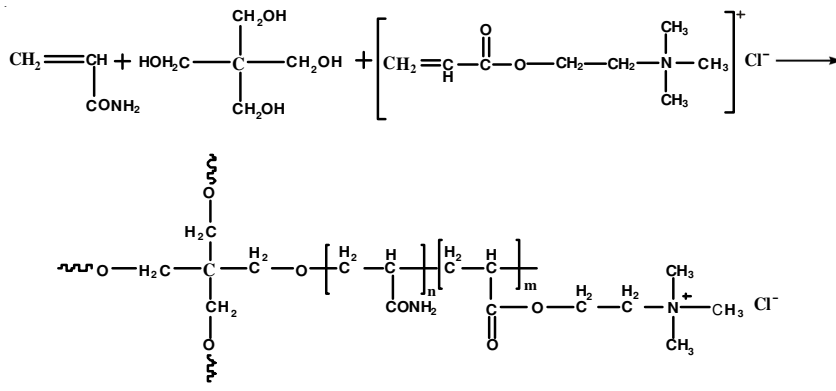

Fig. 1. Synthesis reactions of star-shaped cationic polyacrylamide

FTIR spectroscopy: In order to determine the structure of the star-shaped cationic polyacrylamide, infrared spectroscopy was performed (Fig. 2). The strong peak at $952 \mathrm{~cm}^{-1}$ indicated absorption of the quaternary ammonium salt. The strong peak around $1170 \mathrm{~cm}^{-1}$ could be assigned to an acyloxy group and the band at $1677 \mathrm{~cm}^{-1}$ was an acrylamino group. The absorption peak at $3444 \mathrm{~cm}^{-1}$ was an amino and hydroxygroup. These are consistent with a copolymer of acrylamide and DAC.

${ }^{1}$ H NMR spectroscopy: The molecular structure of the cationic polyacrylamide is shown in Fig. 3. ${ }^{1} \mathrm{H}$ NMR spectroscopy of the star-shaped cationic polyacrylamide solution in $\mathrm{D}_{2} \mathrm{O}$ is shown in Fig. 4.

It can be seen from Fig. 4 that the chemical shift of $\mathrm{H}$ atoms (1) was $3.181 \mathrm{ppm}, \mathrm{H}$ atoms (2) was $3.712 \mathrm{ppm}, \mathrm{H}$ atoms (3) was $4.533 \mathrm{ppm}, \mathrm{H}$ atoms (4) was $1.594 \mathrm{ppm}, \mathrm{H}$ atoms (5) was $2.330 \mathrm{ppm}, \mathrm{H}$ atoms (6) was $2.180 \mathrm{ppm}, \mathrm{H}$ atoms (7) was $1.720 \mathrm{ppm}$ and $\mathrm{H}$ atoms (8) was $4.052 \mathrm{ppm}$.

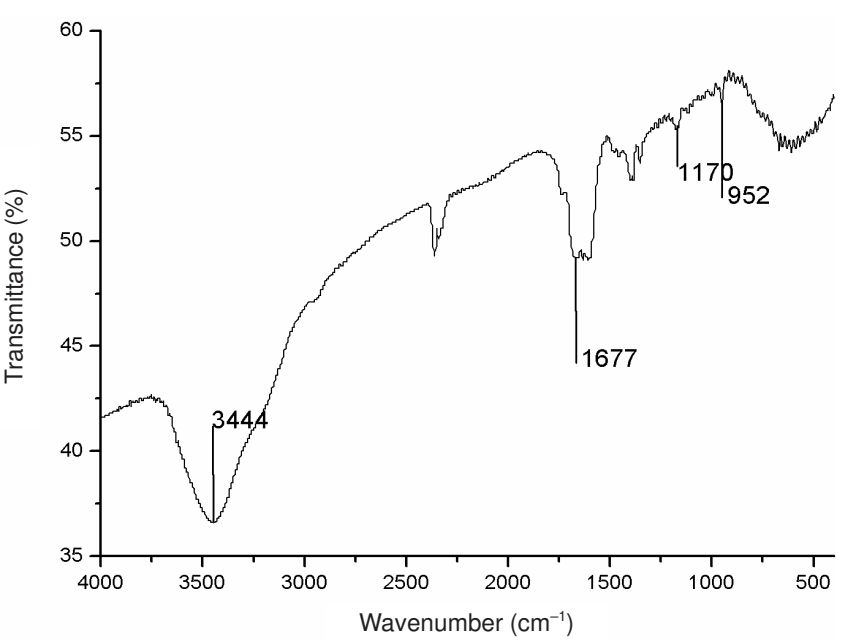

Fig. 2. FTIR of star-shaped cationic polyacrylamide

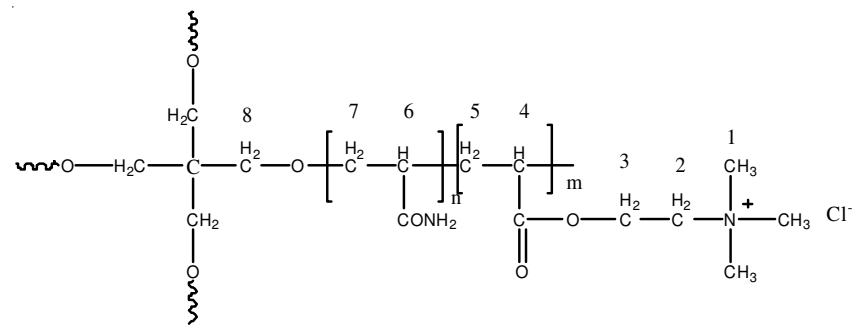

Fig. 3. Molecular structure of star-shaped cationic polyacrylamide

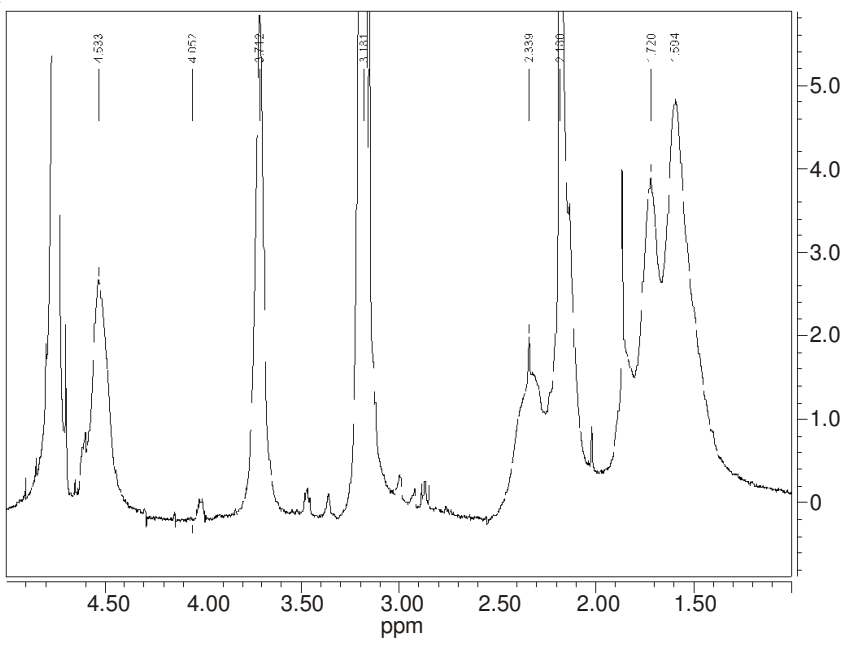

Fig. 4. ${ }^{1} \mathrm{H}$ NMR of star-shaped cationic polyacrylamide

The analytical results of the FTIR and ${ }^{1} \mathrm{H}$ NMR spectroscopy provided support for the formation of a star-shaped cationic polyacrylamide.

Effects of the concentration of monomers: The concentrations of total monomers used were 10, 15, 20, 25, 30 and $35 \%$, respectively. Reactions were performed at $50{ }^{\circ} \mathrm{C}$ for 2.5 $\mathrm{h}$. The mass ratio of acrylamide to DAC was kept at $1: 1$, the amount of ammonium persulfate was $0.025 \%$ of monomers, the ratio of ammonium persulfate to $\mathrm{Na}_{2} \mathrm{SO}_{3}$ was $2: 1$ and the amount of pentaerythritol was 0.1492 times that of ammonium persulfate. The results are shown in Figs. 5 and 6.

Fig. 5 illustrates the relationship between concentration of monomers and rate of production or intrinsic viscosity of the star-shaped cationic polyacrylamide. The intrinsic viscosity 


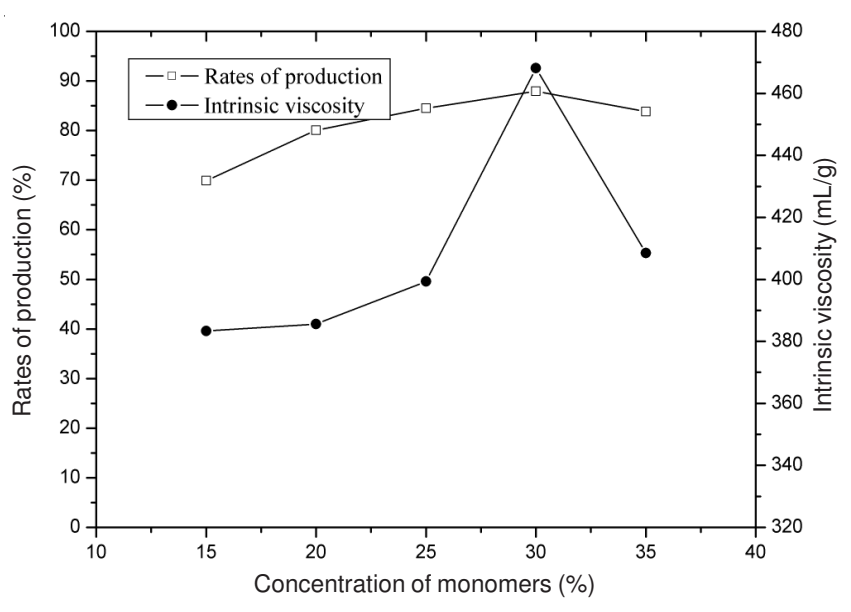

Fig. 5. Effects of concentration of monomers

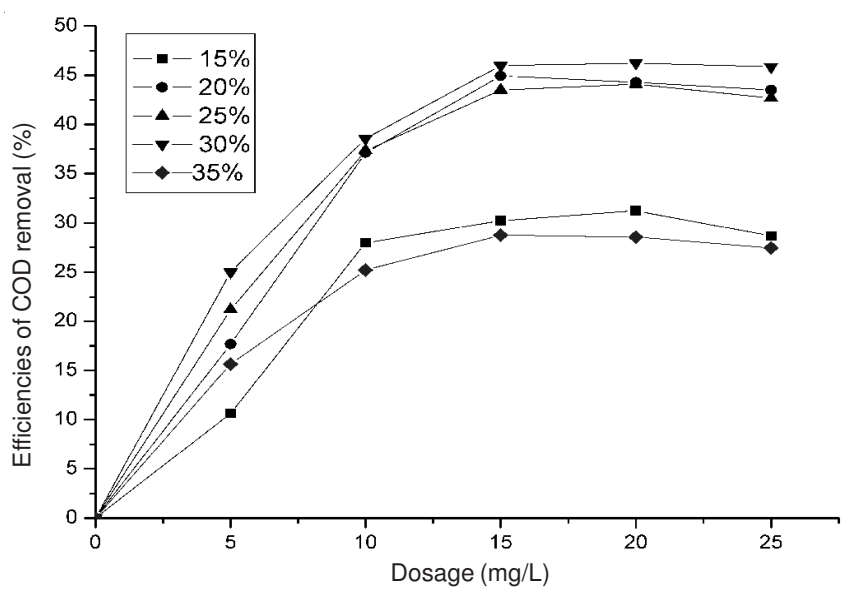

Fig. 6. Effects of efficiency of removal of COD for sewage

of the polymer increased with increasing concentration of monomers, up to a $30 \%$ monomer concentration. At higher monomer concentrations, the high viscosity of the polymer solution inhibited chain propagation, resulting in decreased intrinsic viscosity. The rate of production acted in a similar fashion. Because of the increased chance of monomer collision with increasing monomer concentration, chain growth becomes easier. However, at high monomer concentration, cross-linking occurs. With excessive monomer concentration, the rate of reaction increases, polymer cross-linking is more likely to occur and solubility becomes poor. In contrast, if the monomer concentration is too low, the chance of acrylamide and cationic monomer contact becomes lower and the growth of the polymer chain is inhibited.

Fig. 6 illustrates the flocculating properties of polymers prepared for sewage. The removal efficiency of COD for sewage increased with increasing dosage of polymer. Above $15 \mathrm{mg} / \mathrm{L}, \mathrm{COD}$ removal remained relatively constant. The flocculating properties of star-shaped cationic polyacrylamide prepared using a $30 \%$ total monomer concentration was associated with the best rate of COD removal (Fig. 6).

Effects of concentration of initiators: After the optimum concentration of monomers was determined, the best concentration of initiator was identified. Reaction conditions were a total monomer concentration of $30 \%$, mass ratio of acrylamide to DAC $1: 1$, reaction temperature of $50^{\circ} \mathrm{C}$, ratio of ammonium persulfate to $\mathrm{Na}_{2} \mathrm{SO}_{3} 2: 1$, an amount of pentaerythritol 0.1492 times that of ammonium persulfate and 0.0025, 0.005, 0.0075, 0.01 and $0.0125 \mathrm{~g}$ of ammonium persulfate. The results are shown in Figs. 7 and 8.

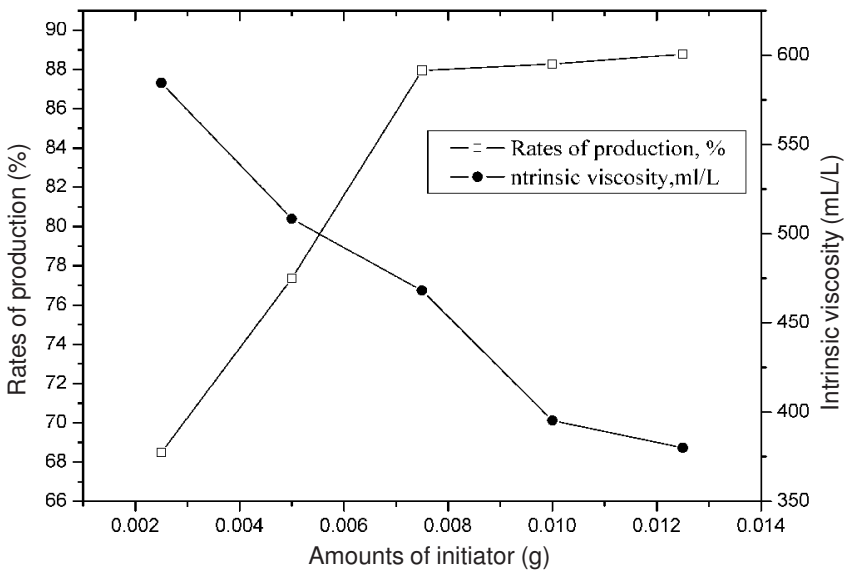

Fig. 7. Effects of concentration of initiator

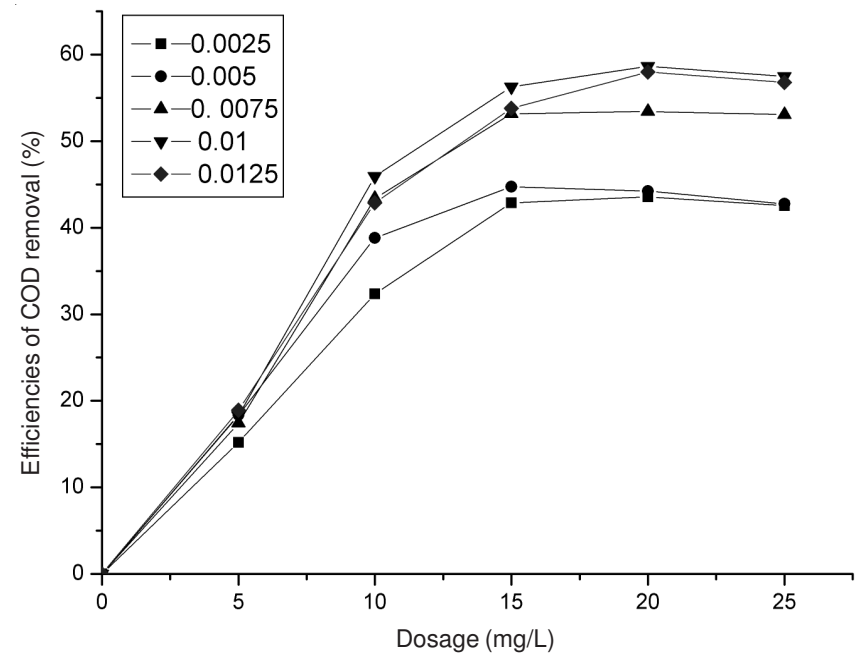

Fig. 8. Effects of efficiency of removal of COD for sewage

Fig. 7 shows the inverse relationship between concentration of initiator and the intrinsic viscosity of the product. The rate of production increased with increasing initiator used, up to $0.0008 \mathrm{~g}$. Above this amount, the rate of production remained relatively constant. In standard polymer reaction kinetics, the more initiator that is used, the more free radical active centers that will be produced and the lower the corresponding polymer molecular weight. The resulting monomer conversion rate is higher. A smaller amount of initiator used is associated with a higher molecular weight copolymer and the monomer conversion rate is lower. If the amount of initiator used is too low, polymerization will not occur. If too much initiator is used, the reaction will heat up quickly and a gel will be produced. Cross-linking of the polymer occurs and the reaction rate is not controllable. If other reaction conditions are constant, decreasing the amount of initiator is associated with longer reaction times, lower conversion rates and higher molecular weight.

Fig. 8 shows the flocculating properties of star-shaped cationic polyacrylamide for sewage. The activity associated 
with different concentrations of initiator used in copolymer production is shown. The removal efficiency of COD for sewage increased with the increasing amount of copolymer used. Doses above $15 \mathrm{mg} / \mathrm{L}$ were not associated with increased COD activity. The best removal efficiency of COD was associated with an initiator amount of $0.010 \mathrm{~g}$. The optimal initiator dose $(0.010 \mathrm{~g})$ was $0.03 \%$ of the total monomer used.

Effects of reaction temperature: In order to investigate the effect of reaction temperature on flocculating properties of polymers produced, the reaction conditions used were $0.03 \mathrm{~g}$ ammonium persulfate, $30 \%$ monomer concentration, ratio of ammonium persulfate to $\mathrm{Na}_{2} \mathrm{SO}_{3} 2: 1$, ratio of acrylamide to DAC $1: 1$ and reaction temperatures of $35,40,45,50$ and $55^{\circ} \mathrm{C}$. The results are shown in Figs. 9 and 10.

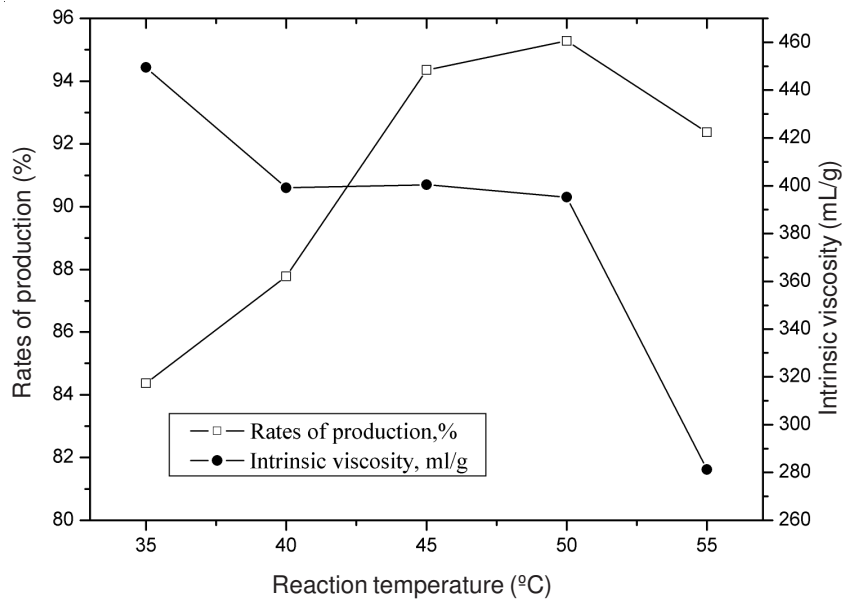

Fig. 9. Effects of reaction temperature

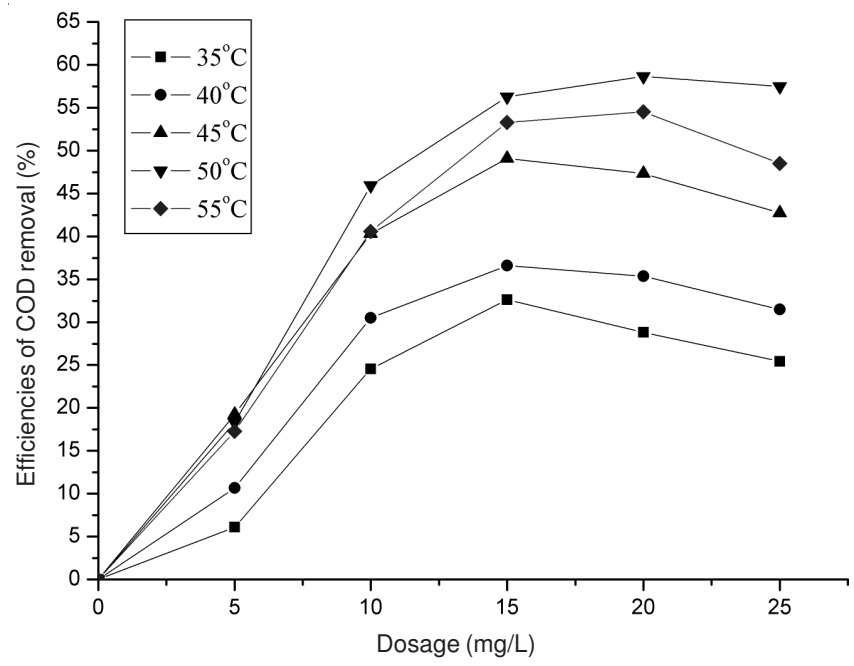

Fig. 10. Effects of efficiency of removal of COD for sewage

Fig. 9 shows the relationship between reaction temperature and intrinsic viscosity or rate of production of cationic polyacrylamide. The intrinsic viscosity of copolymer decreased with increasing reaction temperature. The rate of production increased with increasing temperature, leveled out at $45-50{ }^{\circ} \mathrm{C}$ and then decreased. In standard reaction kinetics, a decrease in reaction temperature will result in lower free radical activity. Resulting polymerization is slower and the rate of propagation is slower. The longer the reaction time, the higher the copolymer molecular weight will be. At higher reaction temperatures, free radicals are more disposed to polymerize and the rate of chain transfer is higher than the rate of chain growth. This leads to more side branching and reduced copolymer quality. If the polymerization reaction is very fast, the solution becomes viscous, free radical and monomer diffusion becomes very slow, and chain propagation is slowed. Higher reaction temperatures can also cause imidization, cross linking, decreased solubility of polymer and decreased rate of polymerization. Given these theoretical considerations for molecular weight and conversion rate, the $50{ }^{\circ} \mathrm{C}$ reaction temperature was the best choice.

Fig. 10 illustrates COD removal efficiencies using different amounts of cationic polyacrylamide. The relationship for each reaction temperature is shown. The efficiency increased to a maximum at $15-20 \mathrm{mg} / \mathrm{L}$ and then decreased with higher amounts. The reaction temperature of $50{ }^{\circ} \mathrm{C}$ was associated with the maximum efficiency of COD removal.

Effects of the ratio of monomers: In order to determine the effect of the mass ratio of acrylamide and DAC on flocculating properties of polymers, the amount of ammonium persulfate was kept constant at $0.01 \mathrm{~g}$, concentration of monomer was kept constant at $30 \%$, a 2:1 ratio of ammonium persulfate to $\mathrm{Na}_{2} \mathrm{SO}_{3}$ was used, reaction temperature was 50 ${ }^{\circ} \mathrm{C}$ and the mass ratio of acrylamide to DAC was varied as $3: 2$, 2:1, 1:1, 1:2 and 2:3. The results are shown in Figs. 11 and 12.

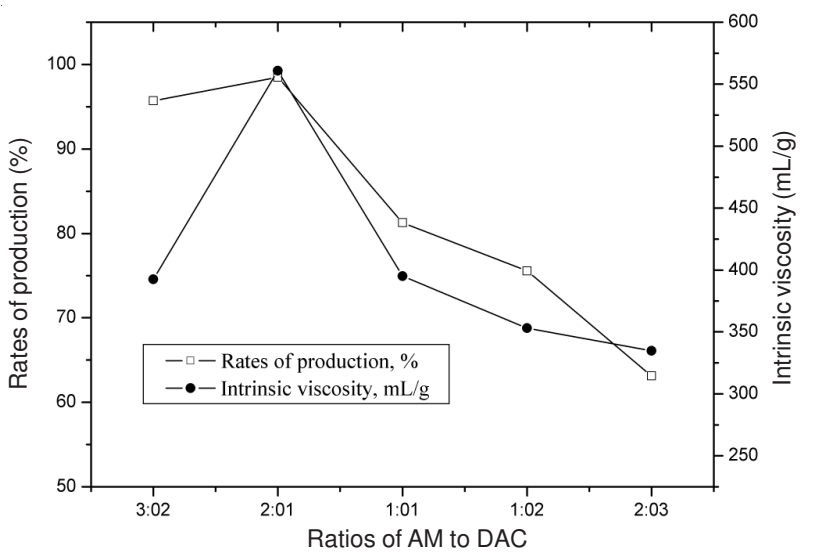

Fig. 11. Effects of concentration of monomers

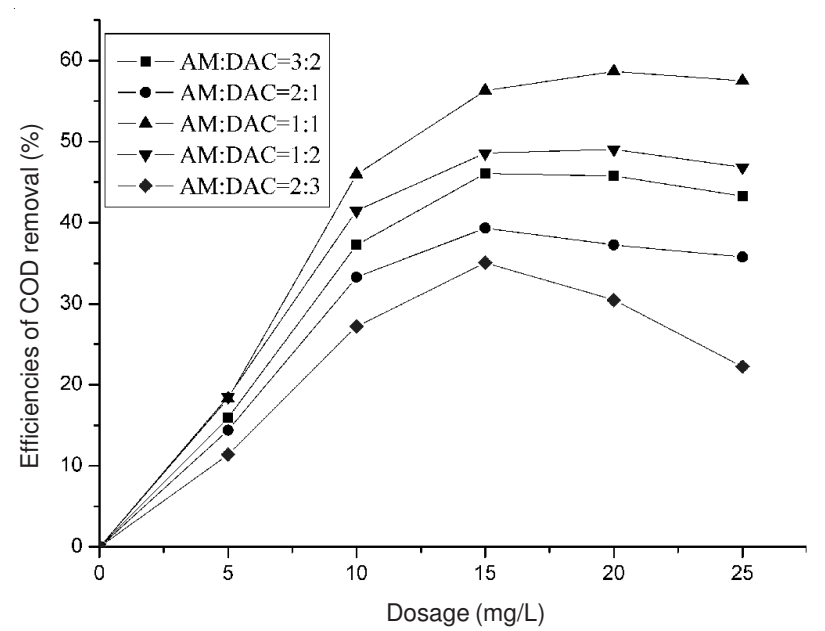

Fig. 12. Effects of efficiency of removal of COD for sewage 
Fig. 11 illustrates the relationship between the mass ratio of monomers, intrinsic viscosity and rate of production of starshaped cationic polyacrylamide. Increasing the ratio of DAC was associated with initial increases in the intrinsic viscosity of the polymer, followed by decreasing viscosity. The rate of production followed a similar pattern. Because there was more DAC present in solution, DAC tended to copolymerize, while acrylamide tended to homopolymerize. The tendency toward copolymerization was greater than homopolymerization. The conversion of DAC was less than that of acrylamide, increasing the content of DAC in any polymer formed and forming more homopolymers. Increased cationic DAC content of the polymer will result in increased polarity of the molecule. This will make removal of water from the product more difficult. The intrinsic viscosity of the copolymer obtained was stable at monomer ratios from $3: 2$ to $1: 1$.

Fig. 12 shows the removal efficiencies of COD for sewage of cationic polyacrylamide as related to amount used. The effect of different acrylamide to DAC ratios during synthesis on COD efficiency is also presented. A dosage of $20 \mathrm{mg} / \mathrm{L}$ appeared optimal for removal efficiency of COD. A 1:1 mass ratio of acrylamide to DAC was associated with the best efficiency of removal of COD.

\section{Conclusion}

The star-shaped cationic polyacrylamide was synthesized using radical copolymerization, with pentaerythritol as the core, acrylamide and acryloxyethyltrimethyl ammonium chloride as monomers and ammonium persulfate and sodium sulfite as the reduction oxidation initiation system. The concentration of initiator was $0.03 \%$ of all monomers, the concentration of monomers was $30 \%$, the reaction temperature was $50{ }^{\circ} \mathrm{C}$ and the optimal mass ratio of acrylamide to DAC at 1:1. Polymer structure was characterized using FTIR and ${ }^{1} \mathrm{H}$ NMR spectrum. The star-shape cationic polyacrylamide was synthesized and successfully used as a novel flocculant in wastewater treatment.

\section{ACKNOWLEDGEMENTS}

Financial support was obtained from the Northwestern Polytechnical University Basic Research Fund (JC20120249).

\section{REFERENCES}

1. American Water Works Association. Water Quality and Treatment - A Handbook of Community Water Supplies, 5th Edition. New York: McGraw-Hill, INC, edn. 5, pp. 6.1-6.2 (1999).

2. J.W. Qian, X.J. Xiang, W.Y. Yang, M. Wang and B.Q. Zheng, Eur. Polym. J., 40, 1699 (2004).

3. F.J. Escudero-Sanz, J.R. Ochoa-Gómez, P.M. Sasia, E.D. de Apodaca and P. Río, J. Appl. Polym. Sci., 103, 2826 (2007).

4. K. Endo and T. Swada, Colloid Polym. Sci., 279, 1058 (2001).

5. M. Daoud and J.P. Cotton, J. Physique, 43, 531 (1982).

6. Ministry of Environmental Protection of the People's Republic of China: Monitoring And Analysis Method of Water and Waste Water China Environmental Science Press, Beijing, edn 4, pp. 210-213 (2002) (in Chinese). 